\title{
Numerical Study of Pressure Fluctuations due to High-Speed Turbulent Boundary Layers
}

\author{
Lian Duan* \\ National Institute of Aerospace, Hampton, VA 23666 \\ Meelan M. Choudhari ${ }^{\dagger}$ \\ NASA Langley Research Center, Hampton, VA 23681 \\ Minwei $\mathrm{Wu}^{\ddagger}$ \\ National Institute of Aerospace, Hampton, VA 23666
}

\begin{abstract}
Direct numerical simulations (DNS) are used to examine the pressure fluctuations generated by fully developed turbulence in supersonic turbulent boundary layers with an emphasis on both pressure fluctuations at the wall and the acoustic fluctuations radiated into the freestream. The wall and freestream pressure fields are first analyzed for a zero pressure gradient boundary layer with Mach 2.5 and Reynolds number based on momentum thickness of approximately 2835. The single and multi-point statistics reported include the wall pressure fluctuation intensities, frequency spectra, space-time correlations, and convection velocities. Single and multi-point statistics of surface pressure fluctuations show good agreement with measured data and previously published simulations of turbulent boundary layers under similar flow conditions. Spectral analysis shows that the acoustic fluctuations outside the boundary layer region have much lower energy content within the high-frequency region. The space-time correlations reflect the convective nature of the pressure field both at the wall and in the freestream, which is characterized by the downstream propagation of pressure-carrying eddies. Relative to those at the wall, the pressure-carrying eddies associated with the freestream signal are larger and convect at a significantly lower speed. The preliminary DNS results of a Mach 6 boundary layer show that the pressure rms in the freestream region is significantly higher than that of the lower Mach number case.
\end{abstract}

\section{Nomenclature}

$C_{p} \quad$ heat capacity at constant pressure, $\mathrm{J} /(\mathrm{K} \cdot \mathrm{kg})$

$C_{v}$ heat capacity at constant volume, $\mathrm{J} /(\mathrm{K} \cdot \mathrm{kg})$

$H$ shape factor, $H=\delta^{*} / \theta$, dimensionless

$M \quad$ Mach number, dimensionless

$\operatorname{Pr} \quad$ Prandtl number, $P r=0.71$, dimensionless

$R e_{\theta}$ Reynolds number based on momentum thickness and freestream viscosity, $R e_{\theta} \equiv \frac{\rho_{\infty} u_{\infty} \theta}{\mu_{\infty}}$, dimensionless

$R e_{\delta 2}$ Reynolds number based on momentum thickness and wall viscosity, $R e_{\delta_{2}} \equiv \frac{\rho_{\infty} u_{\infty} \theta}{\mu_{w}}$, dimensionless

$R e_{\tau}$ Reynolds number based on shear velocity and wall viscosity, $R e_{\tau} \equiv \frac{\rho_{w} u_{\tau} \delta}{\mu_{w}}$, dimensionless

$T$ temperature, $\mathrm{K}$

$T_{r} \quad$ recovery temperature, $T_{r}=T_{\infty}\left(1+0.9 * \frac{\gamma-1}{2} M_{\infty}^{2}\right), \mathrm{K}$

\footnotetext{
${ }^{*}$ Research Scientist. Member, AIAA

${ }^{\dagger}$ Aerospace Technologist, Computational AeroSciences Branch, M.S. 128. Associate Fellow, AIAA

$\ddagger$ Research Scientist.

Copyright (C) 2012 by the American Institute of Aeronautics and Astronautics, Inc. The U.S. Government has a royalty-free license to exercise all rights under the copyright claimed herein for Governmental purposes. All other rights are reserved by the copyright owner.
} 


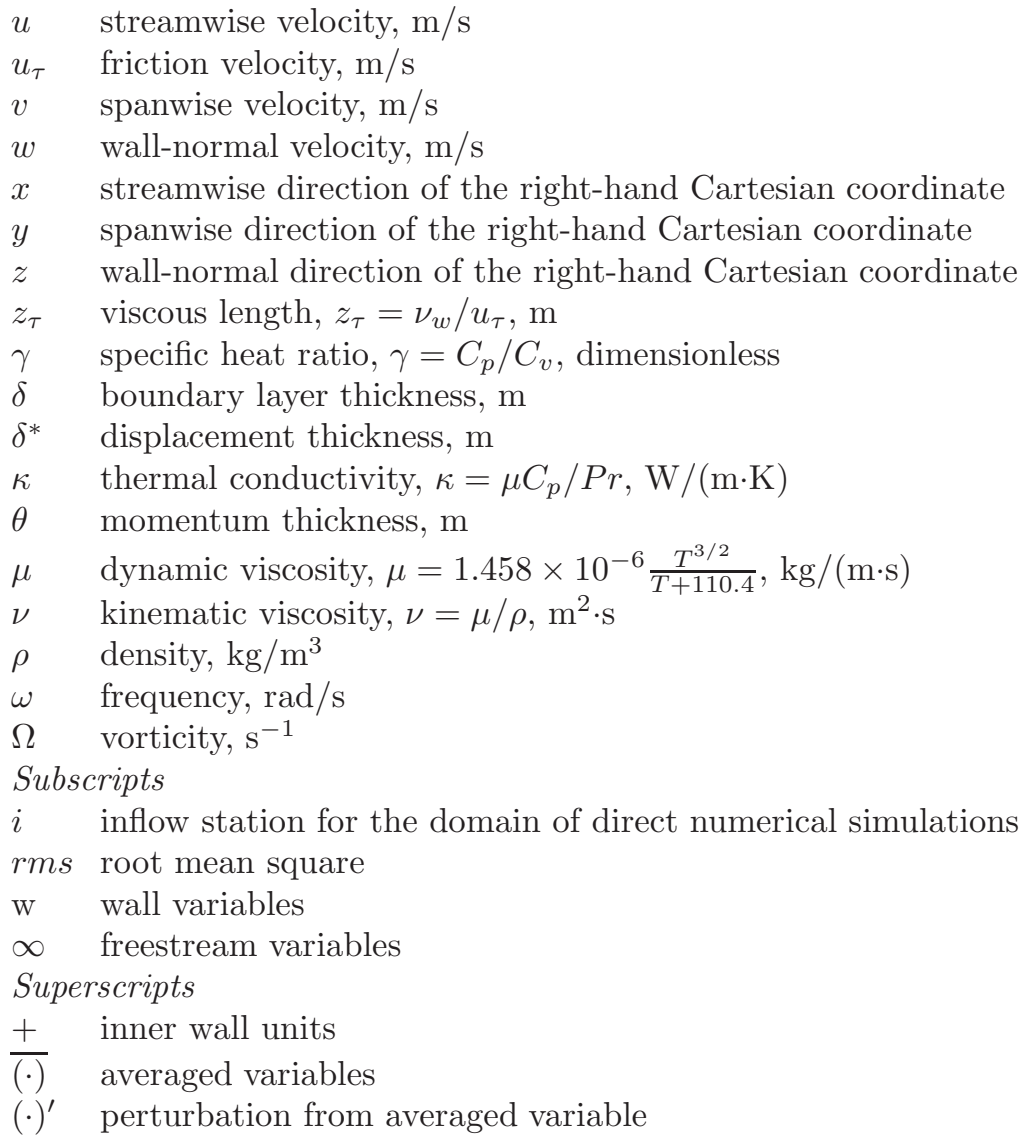

\section{Introduction}

It is well-known that conventional wind tunnel facilities cannot reliably simulate the in-flight transition behavior of supersonic and hypersonic boundary layer flows over a smooth surface. Recent evidence ${ }^{1}$ suggests that the tunnel flow quality also has an impact on the subcritical transition due to isolated surface roughness of sufficiently large height. Over the years, a large body of transition data has been acquired in conventional high-speed facilities despite the potential impact of facility disturbances on the transition onset location and, perhaps, even on the underlying transition mechanism(s). Indeed, even with the advent of low disturbance (i.e. quiet) wind tunnels, the conventional facilities continue to be used today because of the size and Reynolds number limitations of the existing quiet facilities.

The higher amplitude of the free-stream disturbances in a conventional (i.e., noisy) high-speed wind tunnel facility usually leads to an earlier onset of transition relative to that in a flight environment or in a quiet tunnel. However, in cases where the dominant frequencies of the noisy tunnel environment are considerably lower than those of the most amplified boundary layer instabilities, it might be argued that the conventional tunnel could still mimic the transition behavior in a low disturbance environment. Therefore, an in-depth knowledge of the broad-band disturbance environment is essential to reconcile any observed differences in transition onset across multiple facilities.

The free-stream disturbances in a conventional high-speed facility are typically dominated by acoustic radiation from the turbulent tunnel wall boundary layers, ${ }^{2-4}$ in addition to the pressure fluctuations associated with the unsteady displacement of the mean pressure gradient and the temperature spottiness (i.e., entropy fluctuations) resulting from non-uniform heating of the upstream flow. To enable a better use of the transition data from conventional facilities, it is important to understand the acoustic disturbance environment in those facilities. Measurements at Purdue University (Schneider, private communication, 2010) indicate that the effect of tunnel disturbances on transition cannot be quantified in terms of a single metric corresponding to the root-mean-square amplitude of free-stream acoustic disturbances. With increased knowledge of the receptivity mechanisms of high-speed boundary layers, ${ }^{5}$ it becomes even more important to characterize the details of the tunnel acoustics originating from the tunnel-wall turbulent boundary layers. Even during 
flight, the laminar boundary layer flow over the aerodynamic surface of interest may be exposed to acoustic radiation from the turbulent boundary layer over other neighboring surfaces, not unlike the model mounted within a conventional wind tunnel.

Although the surface pressure fluctuations underneath subsonic turbulent boundary-layer flows have been studied extensively over the years, ${ }^{6-8}$ much less attention has been devoted to the pressure fluctuations induced by supersonic and hypersonic wall-bounded flows. The few existing measurements of fluctuating wall pressure signals beneath supersonic turbulent boundary layers include early measurements by Kistler and $\mathrm{Chen}^{9}$ and by Maestrello ${ }^{10}$ for boundary layers with $M_{\infty}$ ranging from 1.33 to 5 , and more recently by Beresh et al. ${ }^{11}$ for boundary layers with $M_{\infty}$ up to 3 . These measurements exhibit a considerable degree of scatter. For example, the measurements by Kister and Chen ${ }^{9}$ and by Maestrello ${ }^{10}$ found discrepancies in the magnitude of wall pressure fluctuations as large as 30\%. The recent data acquired by Beresh et al. showed similar large scatter when compared with a broad compilation of high-speed measurements. As pointed out by several authors, ${ }^{11,12}$ there are few (if any) reliable measurements of the variance of the wall pressure fluctuations and its frequency spectra, due to the poor spatial resolution of pressure transducers or limitations in the frequency response of pressure sensors. As far as the acoustic fluctuations in the freestream region are concerned, the only detailed measurements have been reported by Laufer ${ }^{2}$ and these measurements are subject to similar sources of experimental error as the wall-pressure measurements. Moreover, as noted by Laufer, ${ }^{2}$ the interpretation of disturbance measurements in a wind tunnel is further complicated by the fact that the measurements reflect the combined outcome of acoustic radiation from all sides of the tunnel wall. As a result, highly accurate measurements of the absolute amplitudes of the radiated acoustic energy were not pursued and only the statistical quantities that were least likely to be influenced by the presence of multiple tunnel walls were investigated.

Direct numerical simulation (DNS) is an invaluable tool which can overcome many of the aforementioned difficulties with experimental measurements and provide access to quantities that are difficult to obtain otherwise, although, of course, the simulations have other limitations related to Reynolds number, domain size, and the duration of data sampling. For the study of the freestream pressure field, in particular, DNS adds the additional benefits of isolating more easily the acoustic radiation from a single surface. DNS studies of wall pressure fluctuations beneath supersonic turbulent boundary layers have been successfully demonstrated recently by Bernardini and Pirozzoli ${ }^{13}$ who reported power spectral densities, pressure fluctuation intensities, integral length scales, and convection speeds for freestream Mach numbers of 2, 3, and 4. Numerical studies have not yet been performed for the behavior of pressure fluctuations in the freestream.

This paper is focused on DNS studies of both the surface and freestream pressure fluctuations generated by a supersonic turbulent boundary layer at Mach 2.5, which is relevant to supersonic transports. The flow conditions selected for numerical simulation and the numerical method are outlined in Section II. Section III is focused on an analysis of the surface and freestream pressure fluctuations. Analogous computations for a Mach 6 boundary layer, which falls within the hypersonic regime, are currently in progress and preliminary results on the single point statistics are reported in Section IV. Conclusions thus far are outlined in Section V.

\section{Flow conditions and numerical methodology}

Relevant flow conditions for the Mach 2.5 case are summarized in Table 1. The domain size for computations is $\left(57.2 \delta_{i}, 15.6 \delta_{i}, 41 \delta_{i}\right)$ in the stream-wise, span-wise, and wall-normal directions, respectively, where $\delta_{i}$ represents the boundary layer thickness based on $u / U_{\infty}=0.99$ at the inflow boundary. The number of grid points is $(1760,800,400)$ in the streamwise, spanwise, and wall-normal directions, respectively. Uniform grid spacings are used in the streamwise and spanwise directions with grid spacings $\Delta x^{+}=8.6$ and $\Delta y^{+}=5.2$, respectively, based on the viscous length scale $z_{\tau}$ at the location selected to gather statistics (listed in Table 1). Geometrically stretched wall-normal grids are used within the boundary-layer region with the mesh spacing being approximately $\Delta z^{+}=0.56$ at the wall and $\Delta z^{+}=8.9$ near the boundary layer edge. The computational grid resolution is comparable to those reported in the literature in the context of previous simulations of turbulent wall-bounded flows using comparable numerical algorithms. ${ }^{14-17}$ The adequacy of the domain size is evaluated by monitoring the spatial decay in two-point correlations. To gain further confidence into the accuracy of the numerical simulations, selected measures of first and second order single-point statistics based on the present simulations are compared with established correlations, measured data, and previous numerical simulations.

To simulate the boundary layer flow over the domain, the compressible Navier-Stokes equations are solved 
in generalized curvilinear coordinates. The working fluid is assumed to be an ideal gas with a linear (i.e. Newtonian) stress-strain relation. The Fourier law is used to compute the heat flux terms. A 7th-order weighted essentially non-oscillatory (WENO) scheme ${ }^{18}$ is used to compute the convective flux terms. This particular WENO algorithm combines a high order of accuracy with low dissipation and high bandwidth, which is desirable for time-accurate simulations of compressible turbulence. The resolution properties of this scheme are documented in several references. ${ }^{19,20}$ For the viscous flux terms, a 4 th order central difference scheme is used. The 3rd order low storage Runge-Kutta scheme by Williamson ${ }^{21}$ is used for time integration.

The turbulent inflow is generated using the recycling/rescaling method developed by Xu and Martin ${ }^{22}$ with the recycling station set at $54.6 \delta_{i}$ downstream of the inlet to allow for complete decorrelation between the recycling station and the inflow. Dynamical translation operations ${ }^{23}$ are applied to the recycled turbulence plane at randomly-distributed time intervals to improve low-frequency characteristics of recycling/rescaling inflow turbulence generation.

On the wall, no-slip conditions are applied for the three velocity components and an isothermal condition is used for the temperature with the wall temperature prescribed to be nearly the recovery temperature $T_{r}$. At the top and outlet boundaries, unsteady non-reflecting boundary conditions based on Thompson ${ }^{24}$ are imposed. Periodic boundary conditions are used in the spanwise direction.

For the current spatial simulation, the boundary layer is slowly growing in the streamwise direction, with corresponding variations in $\delta$ from $4 \mathrm{~mm}$ at the inlet to $7.8 \mathrm{~mm}$ near the exit and $R e_{\theta}$ from 1487 to 2865 . Table 1 provides the freestream Mach number, density, and temperature $\left(M_{\infty}, \rho_{\infty}\right.$, and $T_{\infty}$, respectively) as well as the boundary layer thickness and various Reynolds numbers at a selected location $\left(x_{a}=53 \delta_{i}\right.$, where the turbulence statistics are gathered). This selected location is farther enough downstream of the inlet for the memory of the inflow generation to fade out and a nearly uniform acoustic radiation field to to be established. In particular, Figure 1 shows that the pressure fluctuations at the wall and in the freestream have become nearly homogeneous in the streamwise direction after $x / \delta_{i} \approx 35$.

$\begin{array}{cccccccccccc}M_{\infty} & U_{\infty}(\mathrm{m} / \mathrm{s}) & \rho_{\infty}\left(\mathrm{kg} / \mathrm{m}^{3}\right) & T_{\infty}(\mathrm{K}) & T_{w}(\mathrm{~K}) & R e_{\theta} & R e_{\tau} & R e_{\delta 2} & \theta(\mathrm{mm}) & \mathrm{H} & \delta(\mathrm{mm}) & \delta_{i}(\mathrm{~mm}) \\ 2.5 & 823.6 & 0.1 & 270.0 & 568.0 & 2834.8 & 509.9 & 1656.9 & 0.583 & 4.14 & 7.69 & 4.0\end{array}$

Table 1. Boundary layer properties at the station selected for the analysis $\left(x_{a}=53 \delta_{i}\right)$ of the acoustic field for Mach 2.5 DNS.

In the following section, averages are first calculated over a streamwise window of $\left[x_{a}-0.8 \delta_{i}, x_{a}+0.8 \delta_{i}\right]$ and spanwise locations for each instantaneous flow field; then, an ensemble average over flow field snapshots spanning a time interval of more than $570 \delta_{i} / U_{\infty}$ is calculated. To monitor the statistical convergence, flow statistics are computed by averaging over the whole or half the number of the flow-field snapshots and negligible difference $(<1 \%)$ is observed between the two.

The power spectral density (PSD) of the pressure signals has been calculated using the Welch method. ${ }^{25}$ The overall pressure record is subdivided into 8 segments, each including $2 \times 3122$ points. The sampling interval is $\Delta t^{+}=0.27$ and the overall length of each segments is $t_{\text {segment }}^{+}=1670$. Negligible difference in PSD is observed by within the reported frequency range by subdividing the pressure signal into 8 or 12 segments.

\section{Results: Mach 2.5 boundary layer}

The Van-Driest transformed mean velocity profile after the DNS is shown in Figure 2. The mean velocity conforms well to the incompressible law-of-the-wall upon van Driest transformation and shows a (narrow) logarithmic region. Figure 3 shows the root mean square (rms) fluctuations in the three velocity components. The DNS results from Guarini et al. ${ }^{26}$ and Spalart ${ }^{27}$ are plotted for comparison. Good agreement is observed across most of the boundary layer between the current DNS and those of Guarini et al. and Spalart, indicating satisfactory resolution of the boundary layer. 


\section{A. Intensity of wall pressure fluctuations}

The rms pressure profile is shown in Figure 4. The wall pressure rms normalized by the wall shear is about 2.58 and approaches a constant value of 0.385 outside the boundary layer edge. The normalized value of computed rms wall pressure is close to the prediction of $p_{w}^{\prime} / \tau_{w}=3$ based on the model by Bies ${ }^{28}$ and those given by the DNS of Guarini et al, ${ }^{26}$ Spalart, ${ }^{27}$ and Bernadini \& Pirozzoli. ${ }^{13}$ The freestream value is comparable to the experimentally measured value of $p_{w}^{\prime} / \tau_{w} \approx 0.4$ as reported by Laufer. ${ }^{2}$

In addition to the pressure rms, the rms of the velocity components $\left(u_{r m s}^{\prime}, v_{r m s}^{\prime}, v_{r m s}^{\prime}\right)$ and other thermodynamic quantities $\left(\rho_{r m s}^{\prime}, T_{r m s}^{\prime}\right)$ approach constant values (listed in Table 2). It is shown that the fluctuating flow variables in the freestream are small, with values that are at least two orders of magnitude smaller than the mean. Moreover, the rms values of the thermodynamic variables satisfy the isentropic conditions. For example, it can be demonstrated that $p_{r m s}^{\prime} / \bar{p} \approx \gamma \rho_{r m s}^{\prime} / \bar{\rho}$ by using the values listed in Table 2. The latter condition is necessary to establish a purely acoustic field in the freestream. Table 2 also shows the ratio of dilatation variance $\overline{\left(\partial u_{i} / \partial x_{i}\right)^{\prime 2}}$ and vorticity variance $\overline{\Omega_{i}^{\prime} \Omega_{i}^{\prime}}$, which are representative of the acoustic and vorticity mode, respectively. The large value of $\overline{\left(\partial u_{i} / \partial x_{i}\right)^{\prime 2}} / \overline{\Omega_{i}^{\prime} \Omega_{i}^{\prime}}$ gives further evidence that the disturbance field in the freestream is predominantly acoustic.

$\begin{array}{cccccccc}M_{\infty} & u_{r m s}^{\prime} / \bar{u} & v_{r m s}^{\prime} / \bar{u} & w_{r m s}^{\prime} / \bar{u} & p_{r m s}^{\prime} / \bar{p} & \rho_{r m s}^{\prime} / \bar{\rho} & T_{r m s}^{\prime} / \bar{T} & \frac{\overline{\left(\partial u_{i} / \partial x_{i}\right)^{\prime 2}}}{\overline{\bar{R}_{i}^{\prime} \Omega_{i}^{\prime}}} \\ 2.5 & 0.0007560 & 0.0004896 & 0.0008092 & 0.003957 & 0.002824 & 0.001131 & 1799.7\end{array}$

Table 2. The disturbance field in the freestream for the Mach $2.5 \mathrm{DNS}$ at $\operatorname{Re}_{\theta}=2835$.

\section{B. Frequency spectra}

The frequency spectrum of the pressure field is defined as

$$
\Phi_{p}(\omega)=\frac{1}{2 \pi} \int_{-\infty}^{\infty} \overline{p^{\prime}(x, y, z, t) p^{\prime}(x, y, z, t+\tau)} e^{-i \omega \tau} d \tau
$$

Figures $5 \mathrm{a}$ and $5 \mathrm{~b}$ shows the pressure spectrum at the wall and in the freestream. For reference, straight lines with slopes of $-1,-7 / 3$, and -6 are also included to gauge the rate of spectral roll-off across relatively low, mid, and high frequencies, respectively. The general character of the surface pressure spectra is similar to that of low-speed boundary layers, which have been studied extensively over the years (see, for instance, the review by Bull ${ }^{8}$ and the monograph by Blake ${ }^{29}$ ). In particular, the spectrum compares well with the experimental measurements in a low-speed boundary layer by Farabee \& Casarella ${ }^{30}$ and in a Mach 2 supersonic boundary layer by Beresh et al., ${ }^{11}$ as well as with the DNS results by Bernardini \& Pirozzoli. ${ }^{13}$ The DNS wall-pressure spectrum decays weakly as $\omega \rightarrow 0$. The absence of incompressible $\omega^{2}$ scaling at low frequencies in the wall-pressure spectrum is consistent with the supersonic measurements by Beresh et al. ${ }^{11}$ and the DNS by Bernardini \& Pirozzoli. ${ }^{13}$ At high frequencies, the wall-pressure spectrum exhibits a $\omega^{-6}$ scaling, which is similar to the DNS by Bernardini \& Pirozzoli ${ }^{13}$ but steeper than the $\omega^{-5}$ scaling predicted theoretically by Blake. ${ }^{29} \mathrm{~A} \omega^{-n}$ behavior (with $n \approx 0.7-1$ ) corresponding to a universal overlap region ${ }^{31}$ is not apparent in the mid-frequency range for the wall-pressure spectrum, consistent with the DNS results reported by Bernardini \& Pirozzoli. ${ }^{13}$ The absence of the $\omega^{-n}$ behavior (with $n \approx 0.7-1$ ) region may be due to the low values of the Reynolds number in these DNS simulations.

The general features of the freestream spectrum compare well with the measurements by Laufer. ${ }^{2}$ Similar to the wall pressure, the freestream pressure fluctuations also weakly decay as $\omega \rightarrow 0$ and exhibit a $\omega^{-6}$ roll-off at high frequencies. However, it appears to have observable regions of slope comparable to -1 and $-7 / 3$, respectively. Similar behavior for the freestream pressure fluctuations has also been observed by Masutti et al., ${ }^{32}$ who pointed out the acoustic pressure spectrum measured by Laufer ${ }^{2}$ included a prominent region with a frequency dependence of approximately $\omega^{-7 / 3}$. The $-7 / 3$ scaling has been shown to apply to the pressure fluctuations generated by turbulence-turbulence interaction within the inertial subrange of velocity fluctuations in a low speed turbulent flow. ${ }^{33}$ However, the finding that a similar scaling applies to the free-stream acoustic pressure fluctuations that are generated by turbulent fluctuations elsewhere (i.e., 
within the supersonic boundary layer) could not have been anticipated a priori, especially since the surface pressure spectrum does not include a noticeable region corresponding to the $-7 / 3$ scaling. Additional work is necessary to establish the scaling of propagating pressure fluctuations due to interaction of velocity fluctuations from the inertial subrange.

To see the distribution of energy among various scales, Figure 6 shows the scaled pre-multiplied pressure spectrum at the wall and in the freestream. It is shown that the freestream pressure signal has more energy distributed in the lower frequencies with the peak centered at a frequency of $\omega \delta / U_{\infty} \approx 1.45$, which is approximately five times lower than the peak in the pre-multiplied pressure spectrum at the wall. The smaller high-frequency energy content for the radiated pressure spectrum indicates stronger reflection and scattering taking place within the boundary layer, trapping the high-frequency fluctuations.

\section{Space-time correlations}

The statistical properties of the pressure field are investigated through the space-time correlation coefficient defined as

$$
C_{p p}(\Delta x, \Delta y, \Delta t)=\frac{\overline{p^{\prime}(x, y, z, t) p^{\prime}(x+\Delta x, y+\Delta y, z, t+\Delta t)}}{\left(\overline{p^{\prime 2}(x, y, z, t)}\right)^{1 / 2}\left(\overline{p^{\prime 2}(x+\Delta x, y+\Delta y, z, t+\Delta t)}\right)^{1 / 2}}
$$

where $\Delta x$ and $\Delta y$ are spatial separations in the streamwise and spanwise directions, respectively, and $\Delta t$ is the time delay.

Figures $7 \mathrm{a}$ and $7 \mathrm{~b}$ display the contours of streamwise-spanwise correlation $C_{p p}(\Delta x, \Delta y, 0)$ of the pressure fluctuations at the wall and in the freestream, respectively. At both heights, the contours are approximately circular for small separation distances and become stretched in the spanwise direction as the separation distance increases, indicating that the large structures affecting the pressure signal have a greater extent in the span-wise direction than in the streamwise direction. Relative to the wall pressure fluctuations, the extent of coherent acoustic radiation in the freestream is significantly larger in both in-plane directions, indicating more significant impact from the larger structures. The dominance of large structures within the freestream pressure field is consistent with its higher energy content at lower frequencies as indicated in Figure 6.

The space-time correlation contours $C_{p p}(\Delta x, 0, \Delta t)$ of the surface and freestream pressure fluctuations are shown in Figures 8a and 8b, respectively. The shape of the contours at both locations indicates the convective nature of the pressure field, which is characterized by downstream propagation of coherent pressure-carrying eddies. The smaller overall inclination of the correlation contours for the freestream pressure fluctuations indicates that the pressure-carrying eddies at the freestream propagate with a smaller bulk velocity. The somewhat broader shape of contours in Figure $8 \mathrm{~b}$ reflects a wider range of convection speeds of the freestream pressure signal, which in turn indicates its dependence on eddies with a greater disparity in sizes.

To further demonstrate the convective nature of the pressure signal as well as its scale dependence, Figure 9 plots the convection velocity as a function of frequency. The convective velocity is computed using the space-time correlation coefficient $C_{p p}(\Delta x, 0, \Delta t)$ following a similar procedure as Bernadini and Pirozzoli. ${ }^{13}$ For a given time delay $\Delta t$ (corresponding to a frequency $\omega=2 \pi / \Delta t$ ), the convection velocity $U_{c}$ is defined as the ratio $\Delta x_{1} / \Delta t$ taken at the value of $\Delta x_{1}$ where a local maximum of space-time correlation coefficient is attained. For the wall pressure signal, the large-scale disturbances (associated with lower frequencies) convect at $U_{c} \approx 0.8 U_{\infty}$ while the small-scale disturbances convect at $U_{c} \approx 0.6 U_{\infty}$, consistent with the values reported by Bernadini and Pirozzoli ${ }^{13}$ for a Mach 2 turbulent boundary layer. Compared to the wall signal, the pressure-carrying eddies for the freestream signal propagate at significantly smaller speeds with $U_{c} \approx 0.4 U_{\infty}$ for large-scale disturbances and $U_{c} \approx 0.32 U_{\infty}$ for $\omega \delta / U_{\infty}>70$. From the standpoint of transition analysis, the convection velocity is an important characteristic of the stochastic acoustic field in the freestream, since the receptivity characteristics are known to be sensitive to the orientation of the plane wave disturbance. ${ }^{5}$

The freestream convection velocity can be further studied by assuming the acoustic disturbances in the freestream to consist of plane waves. Laufer ${ }^{2}$ shows that the wave angle $\theta$, which is the angle between the flow direction and normal to the wave front (shown in Figure 10), can be determined by

$$
\cos \theta=\gamma M_{\infty} \frac{u^{\prime} / U_{\infty}}{p^{\prime} / p_{\infty}}
$$


Once $\theta$ is known, the velocity of sound sources, $U_{b}$, that produce the waves can be calculated by

$$
\frac{U_{b}}{U_{\infty}}=1+\frac{1}{M_{\infty} \cos \theta}
$$

The wave angle is approximately $\theta \approx 132^{\circ}$ based on Equation 3 . The calculated wave-front orientation is roughly consistent with the inclination of wave fronts from the numerical Schlieren image in Figure 10. The corresponding convection velocity for 'effective' radiation sources is $U_{b} \approx 0.4 U_{\infty}$ according to Equation 4 , and this calculated convection velocity is very close to the convection velocity calculated based on the space-time correlation (Figure 9).

The fact that the freestream pressure disturbance convects at a significantly reduced speed than those at the wall can be explained by the 'eddy Mach wave' concept first proposed by Phillips, ${ }^{34}$ which states that the 'Mach wave type' radiation is produced by eddies which convected supersonically with respect to the freestream. According to the conjecture, the pressure fluctuations at the freestream are predominantly produced by the small-scale, slowly moving eddies whose phase velocity is significantly smaller than the freestream value so that a supersonic relative speed is achieved, while the wall pressure fluctuations are presumed to be produced mainly by the large-scale, energy containing motions with phase velocities closer to that of the freestream.

\section{Mach 6 boundary layer}

Preliminary results for the Mach 6 boundary layer are presented in this section. The Mach 6 case serves to highlight the variation of acoustic characteristics with Mach number as well as to provide additional validation of simulations against experimentally measured trends. In particular, the effects of Mach number on directional characteristics of the freestream acoustic radiation will be demonstrated, since theoretical predictions of boundary-layer receptivity have shown that the streamwise phase velocity, or equivalently, the orientation of a plane-wave acoustic disturbance can have a substantial influence on the amplitudes of the acoustically excited instability waves within a high-speed boundary layer.

For the Mach 6 case, the domain size for computations is $\left(61.4 \delta_{i}, 15.6 \delta_{i}, 41.5 \delta_{i}\right)$ in the stream-wise, span-wise, and wall-normal directions, respectively. The number of grid points is $(1600,800,500)$ in the streamwise, spanwise, and wall-normal directions, respectively. Similar to the Mach 2.5 case, uniform grid spacings are used in the streamwise and spanwise directions with grid spacings $\Delta x^{+}=9.7$ and $\Delta y^{+}=5.2$, respectively, and geometrically stretched grids are used in the wall-normal direction with the mesh spacing being $\Delta z^{+}=0.52$ at the wall and $\Delta z^{+}=5.3$ near the boundary layer edge. Table 3 provides the flow conditions and boundary layer properties at a selected location $x_{a}=59 \delta_{i}$ where turbulence statistics are gathered. Again, this selected location is farther enough downstream of the inlet for the memory of the inow generation to fade out and a nearly uniform acoustic radiation eld to to be established. Figure 11 shows that at the selected Reynolds numbers the Van-Driest transformed mean velocity profile from the Mach 6 DNS includes a (narrow) logarithmic region.

To illustrate the effect of Mach number on directional characteristics of the freestream acoustic radiation, Figure 12 shows the numerical Schlieren image for the Mach 6 case. Similar to the Mach 2.5 case, the acoustic radiation in the freestream is clearly observable. However, the acoustic wave consists of a considerably shallower orientation of the wave fronts with respect to the flow direction $\left(\theta \approx 114^{\circ}\right)$ than the Mach 2.5 boundary layer. The corresponding convection velocity for Mach 6 is $U_{b} \approx 0.59$ based on Equation 4 , which is significantly larger than the value of the Mach 2.5 case. Although not shown, the magnitude of the scaled pressure rms $p_{r m s}^{\prime} / \tau_{w}$ for the Mach 6 case is nearly twice as large as that for the Mach 2.5 case, which is again consistent with the measured trend by Laufer. ${ }^{2}$ A thorough analysis of this data set is still in progress and the results thereof will be presented in a follow-on paper.

The smaller wave angle and increased radiation intensity for the boundary layer with higher freestream Mach number are consistent with the experimentally measured trends ${ }^{2}$ and provides further evidence for the 'eddy Mach wave' hypothesis. At low supersonic freestream Mach numbers, sources that contribute primarily to the radiation field are slowly moving ones, the convection velocities of which are supersonic relative to the freestream. As the Mach number increases, additional faster moving turbulent eddies acquire supersonic relative speeds and start to take part in the radiation process, acounting for the increased overall convection velocity and larger acoustic amplitudes in the freestream. 


$\begin{array}{cccccccccccc}M_{\delta} & U_{\infty}(\mathrm{m} / \mathrm{s}) & \rho_{\infty}\left(\mathrm{kg} / \mathrm{m}^{3}\right) & T_{\infty}(\mathrm{K}) & T_{w}(\mathrm{~K}) & R e_{\theta} & R e_{\tau} & R e_{\delta 2} & \theta(\mathrm{mm}) & \mathrm{H} & \delta(\mathrm{mm}) & \delta_{i}(\mathrm{~mm}) \\ 6.0 & 871.9 & 0.039 & 52.6 & 300.1 & 9540.0 & 516.3 & 1761.3 & 0.00096 & 13.2 & 26.9 & 13.2\end{array}$

Table 3. Boundary layer properties at the station selected for the analysis $\left(x_{a}=59 \delta_{i}\right)$ of the acoustic field for Mach 6 DNS.

\section{Conclusions}

DNS are used to examine the pressure fluctuations generated by fully developed turbulence in supersonic turbulent boundary layers. It is found that for a Mach 2.5 boundary layer the statistical characteristics of the pressure fluctuations, including the fluctuation intensities, frequency spectra, space-time correlations, and convection velocities, show good agreement with measured data and previously published simulations of turbulent boundary layers under similar flow conditions. In particular, the DNS shows that the acoustic fluctuations outside the boundary layer region have much lower energy content at high frequencies, with the peak of the spectrum centered at a frequency approximately five times lower than that associated with the turbulent motions. The pressure field both at the wall and in the freestream are the convective in nature, being characterized by the downstream propagation of pressure-carrying eddies. Relative to those at the wall, the pressure-carrying eddies associated with the freestream signal are larger and convect at a significantly lower speed. The preliminary DNS results of a Mach 6 boundary layer show increased radiation intensity, shallower orientation of wave fronts with respect to the flow direction, and larger convection velocity relative to the Mach 2.5 case. Such variations in the freestream pressure field with Mach number is consistent with the 'Mach wave radiation' concept. ${ }^{34}$

Ongoing work includes an additional quantification of numerical accuracy via grid convergence studies, a focus on the hypersonic Mach number regime (where even the properties of surface pressure fluctuations have not been quantified yet), and an in-depth data analysis and application to boundary-layer transition.

\section{Acknowledgments}

This work was performed as part of the Supersonics Project of NASA's Fundamental Aeronautics Program (FAP). The authors would like to thank Prof. Pino Martín of the University of Maryland for providing the original code which has been modified for the current study. Technical discussions with Robert Rubinstein and Elizabeth Lee-Rausch at NASA Langley Research Center are also acknowledged. The simulations have been conducted using the Pleiades Supercomputer of the NASA Advanced Supercomputing (NAS) Division.

\section{References}

\footnotetext{
${ }^{1}$ Borg, M. P. and Schneider, S. P., "Effect of Free-stream Noise on Roughness-Induced Transition for the X-51A Forebody," Journal of Spacecraft and Rockets, Vol. 45, No. 6, 2008, pp. 1106-1116.

${ }^{2}$ Laufer, J., "Some Statistical Properties of the Pressure Field Radiated by a Turbulent Boundary Layer," Physics of Fluids, Vol. 7, No. 8, 1964, pp. 1191-1197.

${ }^{3}$ Bushnell, D. M., "Notes on Initial Disturbance Fields for the Transition Problem," Instability and Transition, edited by M. Y. Hussaini and R. G. Voigt, Springer-Verlag, Berlin, Vol. 1, 1990, pp. 217-232.

${ }^{4}$ Stetson, K. F., "Nosetip Bluntness Effects on Cone Frustrum Boundary-Layer Transition in Hypersonic Flow," AIAA Paper 83-1763, 1983.

${ }^{5}$ Fedorov, A. V., "Receptivity of a High-Speed Boundary Layer to Acoustic Disturbances," Journal of Fluid Mechanics, Vol. 491, 2003, pp. 101-129.

${ }^{6}$ Willmarth, W. W., "Wall Pressure Fluctuations in a Turbulent Boundary Layer," Journal of Acoustical Society America, Vol. 28, No. 6, 1956, pp. 1048-1053.

${ }^{7}$ Willmarth, W. W., "Wall Pressure Fluctuations beneath Turbulent Boundary Layers," Annual Review of Fluid Mechanics, Vol. 7, 1975, pp. 13-36.

${ }^{8}$ Bull, M. K., "Wall-Pressure Fluctuations beneath Turbulent Boundary Layers: Some Reflection on Forty Years of Research," Journal of Sound and Vibration, Vol. 190, No. 3, 1996, pp. 299-315.

${ }^{9}$ Kistler, A. L. and Chen, W. S., "The Fluctuating Pressure Field in a Supersonic Turbulent Boundary Layer," Journal of Fluid Mechanics, Vol. 16, 1963, pp. 41-64.
} 
${ }^{10}$ Maestrello, L., "Radiation from and Panel Response to a Supersonic Turbulent Boundary Layer," Journal of Sound Vibration, Vol. 10, No. 2, 1969, pp. 261-262.

${ }^{11}$ Beresh, S. J., Henfling, J. F., Spillers, R. W., and Pruett, B. O. M., "Fluctuating Wall Pressures Measured beneath a Supersonic Turbulent Boundary Layer," Physics of Fluids, Vol. 23, No. 7, 2011, 075110.

${ }^{12}$ Dolling, D. S. and Dussauge, J. P., "A survey of measurements and measuring techniques in rapidly distorted compressible turbulent boundary layers," AGARDograph, Vol. 315, 1989, pp. 1-18.

${ }^{13}$ Bernardini, M. and Pirozzoli, S., "Wall Pressure Fluctuations beneath Supersonic Turbulent Boundary Layers," Physics of Fluids, Vol. 23, No. 8, 2011, 085102.

${ }^{14}$ Martín, M., "DNS of Hypersonic Turbulent Boundary Layers. Part I: Initialization and Comparison with Experiments," Journal of Fluid Mechanics, Vol. 570, 2007, pp. 347-364.

${ }^{15}$ Duan, L., Beekman, I., and Martín, M. P., "Direct Numerical Simulation of Hypersonic Turbulent Boundary Layers. Part 3: Effect of Mach Number," Journal of Fluid Mechanics, Vol. 672, 2011, pp. 245-267.

${ }^{16}$ Duan, L., Beekman, I., and Martín, M. P., "Direct Numerical Simulation of Hypersonic Turbulent Boundary Layers. Part 2: Effect of Wall Temperature," Journal of Fluid Mechanics, Vol. 655, 2010, pp. 419-445.

${ }^{17}$ Duan, L. and Martín, M. P., "Direct Numerical Simulation of Hypersonic Turbulent Boundary Layers. Part 4: Effect of High Enthalpy," Journal of Fluid Mechanics, Vol. 684, 2011, pp. 25-59.

${ }^{18}$ Jiang, G. S. and Shu, C. W., "Efficient Implementation of Weighted ENO Schemes," Journal of Computational Physics, Vol. 126, No. 1, 1996, pp. 202-228.

${ }^{19}$ Martín, M. P., Taylor, E. M., Wu, M., and Weirs, V. G., "A Bandwidth-Optimized WENO Scheme for the Direct Numerical Simulation of Compressible Turbulence," Journal of Computational Physics, Vol. 220, No. 1, 2006, pp. $270-289$.

${ }^{20}$ Taylor, E. M., Wu, M., and Martín, M. P., "Optimization of Nonlinear Error Sources for Weighted Non-Oscillatory Methods in Direct Numerical Simulations of Compressible Turbulence," Journal of Computational Physics, Vol. 223, No. 1, 2006, pp. 384-397.

${ }^{21}$ Williamson, J., "Low-Storage Runge-Kutta Schemes," Journal of Computational Physics, Vol. 35, No. 1, 1980, pp. 48-56.

${ }^{22} \mathrm{Xu}$, S. and Martín, M. P., "Assessment of Inflow Boundary Conditions for Compressible Turbulent Boundary Layers," Physics of Fluids, Vol. 16, No. 7, 2004, pp. 2623-2639.

${ }^{23}$ Morgan, B., Larsson, J., Kawai, S., and Lele, S. K., "Improving Low-Frequency Characteristics of Recycling/Rescaling Inflow Turbulence Generation," AIAA Journal, Vol. 49, No. 3, 2011, pp. 582-597.

${ }^{24}$ Thompson, K. W., "Time Dependent Boundary Conditions for Hyperbolic Systems," Journal of Computational Physics, Vol. 68, No. 1, Jan. 1987, pp. 1-24.

${ }^{25}$ Welch, P. D., "The Use of Fast Fourier Transform for the Estimation of Power Spectra: A Method Based on Time Averaging Over Short, Modified Periodograms," IEEE Trans. Audio Electroacoustics, Vol. AU-15, 1967, pp. 70-73.

${ }^{26}$ Guarini, S. E., Moser, R. D., Shariff, K., and Wray, A., "Direct Numerical Simulation of a Supersonic Turbulent Boundary Layer at Mach 2.5," Journal of Fluid Mechanics, Vol. 414, 2000, pp. 1-33.

${ }^{27}$ Spalart, P. R., "Direct Simulation of a Turbulent Boundary Layer up to Re $e_{\theta}=1410$," Journal of Fluid Mechanics, Vol. 187, 1988, pp. 61-98.

${ }^{28}$ Bies, D. W., "A Review of Flight and Wind Tunnel Measurements of Boundary Layer Pressure Fluctuations and Induced Structure Reponse," NASA CR-626, 1966.

${ }^{29}$ Blake, W. K., "Mechanics of Flow-Induced Sound and Vibration," Academic Press, Orlando, Florida, 1986.

${ }^{30}$ Farabee, T. and Casarella, M. J., "Spectral Features of Wall Pressure Fluctuations Beneath Turbulent Boundary Layers," Physics of Fluids, Vol. 3, 1991, pp. 2410-2420.

${ }^{31}$ Bradshaw, P., "Inactive Motion and Pressure Fluctuations in Turbulent Boundary Layers," Journal of Fluid Mechanics, Vol. 30, 1967, pp. 241-258.

${ }^{32}$ Masutti, M., Chazot, E., and Carbo, M., "Disturbance Level Characterization of a Hypersonic Blow-Down Facility," To appear in AIAA Journal, 2012.

${ }^{33}$ George, W. K., Beuther, P. D., and Arndt, R. E. A., "Pressure Spectra in Turbulent Free Shear Flows," Journal of Fluid Mechanics, Vol. 148, 1984, pp. 155-191.

${ }^{34}$ Phillips, O. M., "On the Generation of Sound by Supersonic Turbulent Shear Layers," Journal of Fluid Mechanics, Vol. 9, 1960, pp. 1-28. 


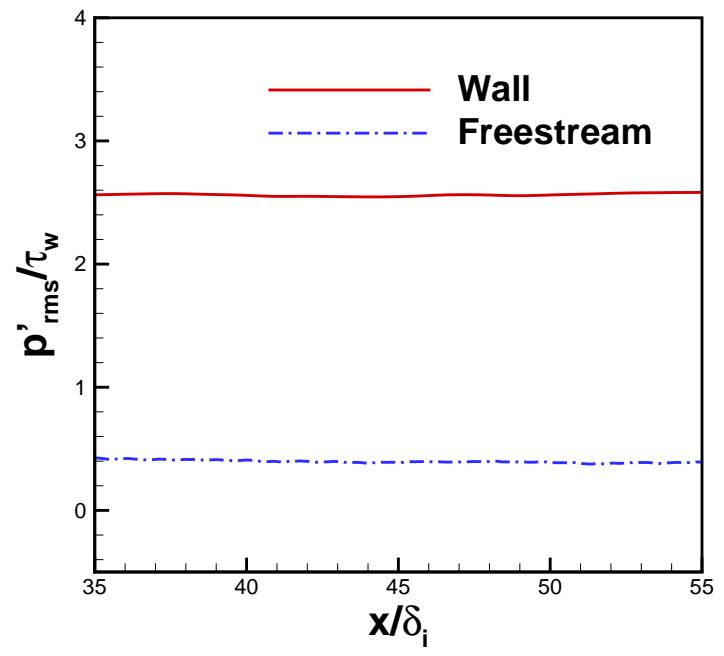

Figure 1. The streamwise distribution of the normalized pressure fluctuation rms at the wall $(z / \delta=0)$ and in the freestream $(z / \delta=2.8)$ for the Mach 2.5 DNS.

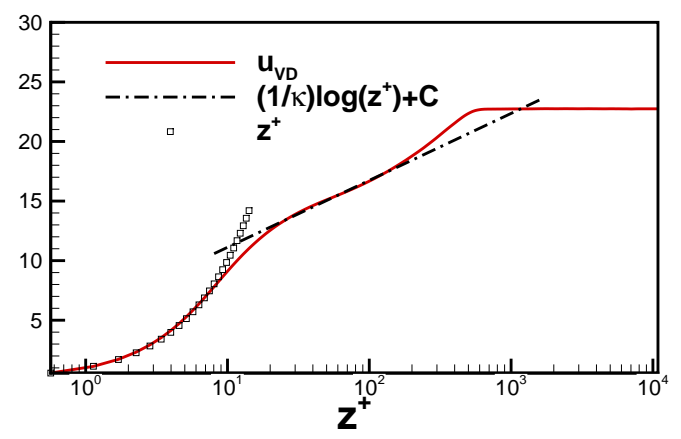

Figure 2. Van-Driest transformed mean velocity profile for the Mach 2.5 DNS $((\kappa=0.41, C=5.5)$. 


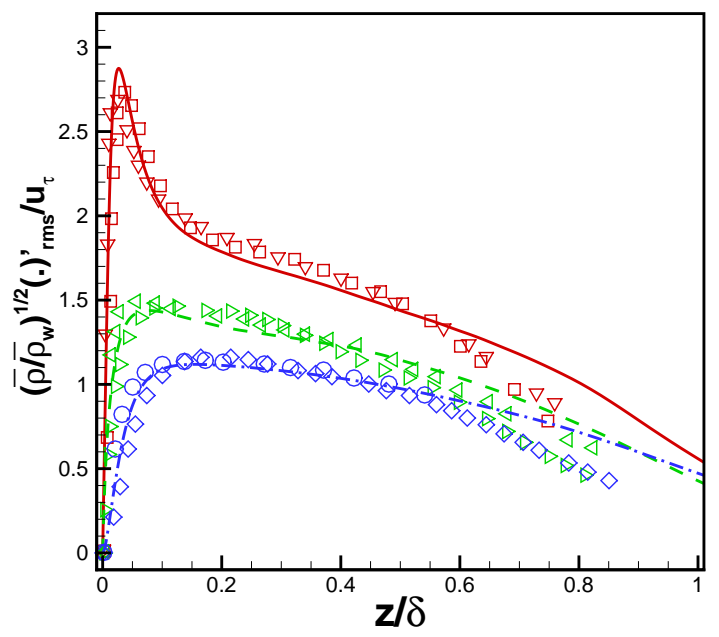

Figure 3. RMS of velocity components for the Mach 2.5 DNS. Streamwise: —_ Current DNS, $\mathrm{M}_{\infty}=2.5, \mathbf{R e}_{\theta}=2835 ; \square$ Guarini et al., ${ }^{26} \mathrm{M}_{\infty}=2.5, \mathbf{R e}_{\theta}=1577 ; \nabla$ Spalart ${ }^{27} \mathbf{M}_{\infty} \approx 0, \mathbf{R e}_{\theta}=1410$. Spanwise: --- Current DNS, $M_{\infty}=2.5, \operatorname{Re}_{\theta}=2835 ; \triangleright$ Guarini et al. ${ }^{26} \mathrm{M}_{\infty}=2.5, \operatorname{Re}_{\theta}=1577$; $\triangleleft$ Spalart, ${ }^{27} \mathrm{M}_{\infty} \approx 0, \mathbf{R e}_{\theta}=1410$. Wall-normal: - - Current DNS, $\mathbf{M}_{\infty}=2.5, \mathbf{R e}_{\theta}=\mathbf{2 8 3 5} ; \diamond$ Guarini et al., ${ }^{26} \mathrm{M}_{\infty}=2.5, \mathrm{Re}_{\theta}=1577$; $\circ$ Spalart, ${ }^{27} \mathrm{M}_{\infty} \approx 0, \mathrm{Re}_{\theta}=1410$.

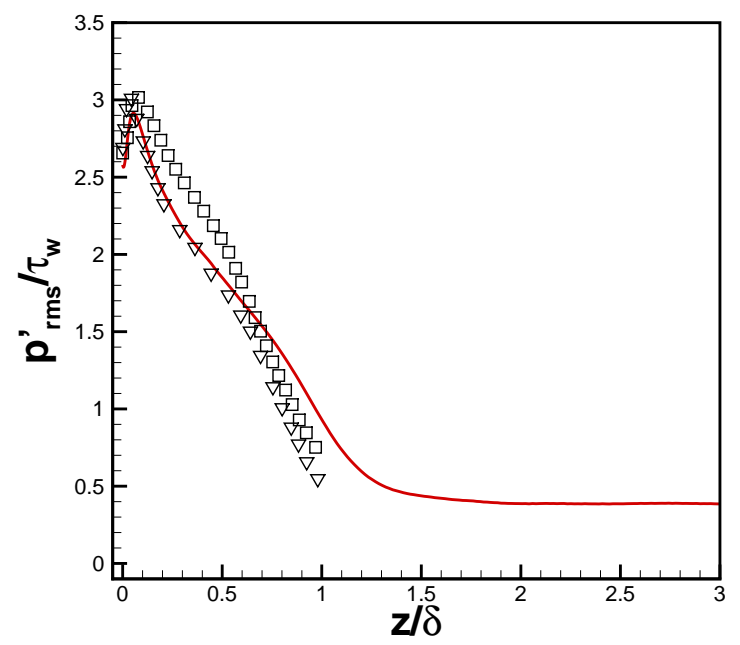

Figure 4. Pressure fluctuation rms profile for the Mach 2.5 DNS. - - Current DNS, $\mathbf{M}_{\infty}=2.5$, $\operatorname{Re}_{\theta}=2835 ; \square$ : Guarini et al., ${ }^{26} \mathrm{M}_{\infty}=2.5, \operatorname{Re}_{\theta}=1577 ; \nabla:$ Spalart, ${ }^{27} \mathrm{M}_{\infty} \approx 0, \operatorname{Re}_{\theta}=1410$. 


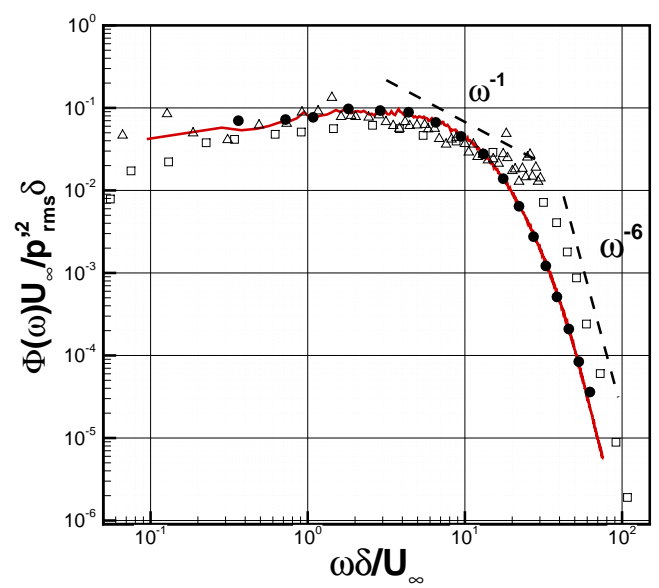

(a) Wall

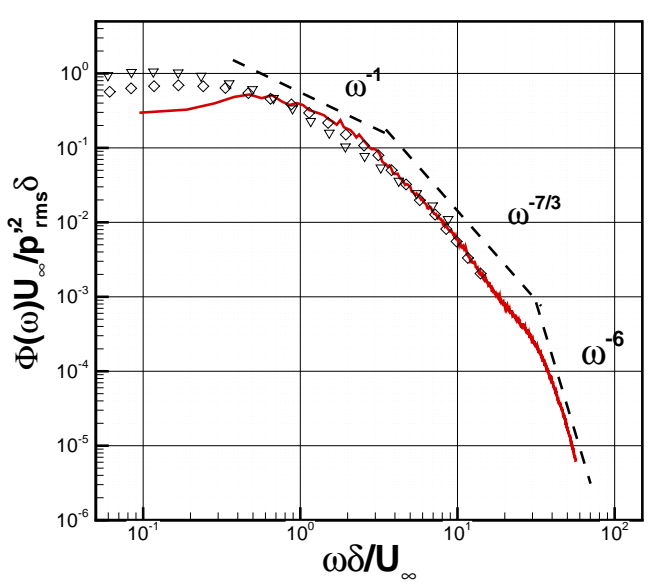

(b) Freestream

Figure 5. Frequency Spectrum of computed pressure signal at selected heights for the Mach 2.5 DNS. - $:$ Current DNS, $\mathbf{M}_{\infty}=2.5, \mathbf{R e}_{\tau}=510 ; \square$ : Farabee and Casarella, ${ }^{30} \mathbf{M}_{\infty} \approx 0$, $\operatorname{Re}_{\tau}=1169 ; \triangle$ : Beresh et al. ${ }^{11} \mathrm{M}_{\infty}=2, \mathbf{R e}_{\tau}=3650 ; \bullet:$ Bernardini and Pirozzoli, ${ }^{13} \mathrm{M}_{\infty}=2$, $\mathbf{R e}_{\tau}=508 ; \nabla:$ Laufer, ${ }^{2} M_{\infty}=2, \operatorname{Re}_{\theta}=30000 ; \diamond:$ Laufer, ${ }^{2} M_{\infty}=4.5, \operatorname{Re}_{\theta}=30000$.

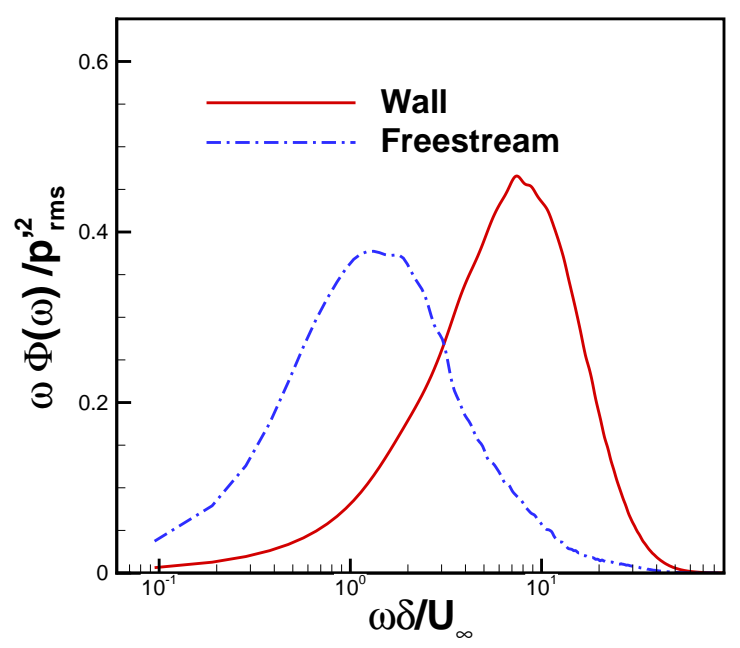

Figure 6. Pre-multiplied power spectrum of pressure signals at at selected heights for the Mach 2.5 DNS. The pressure spectrum is normalized so that the area under each curve is equal to unity. 


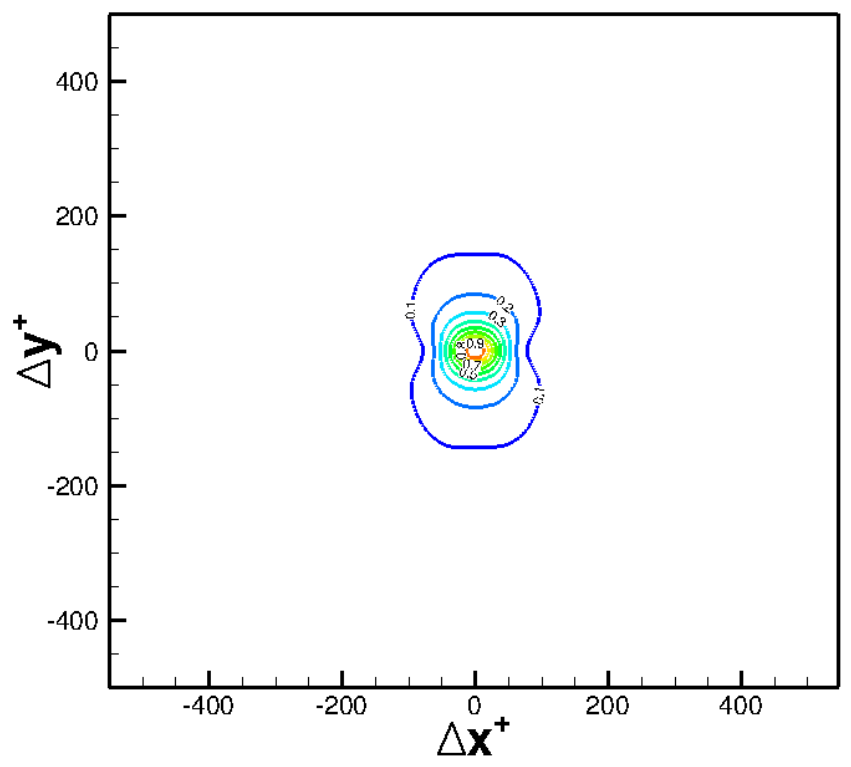

(a) Wall

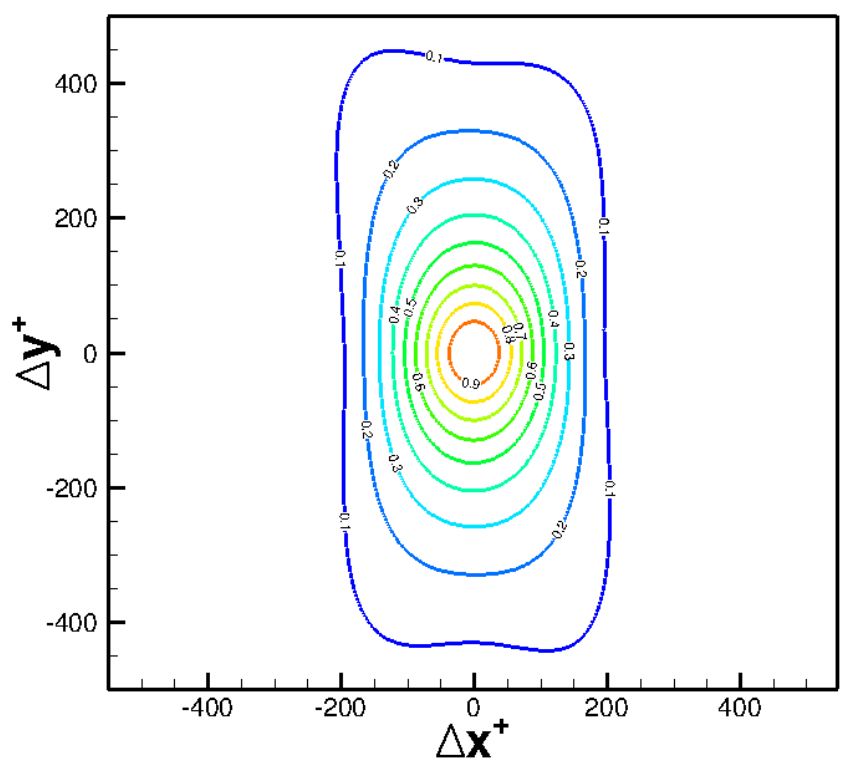

(b) Freestream

Figure 7. Streamwise-spanwise correlation coefficient of the pressure signal at selected heights for the Mach 2.5 DNS. Contour levels vary from 0.1 to 0.9 with increments of 0.1 . 


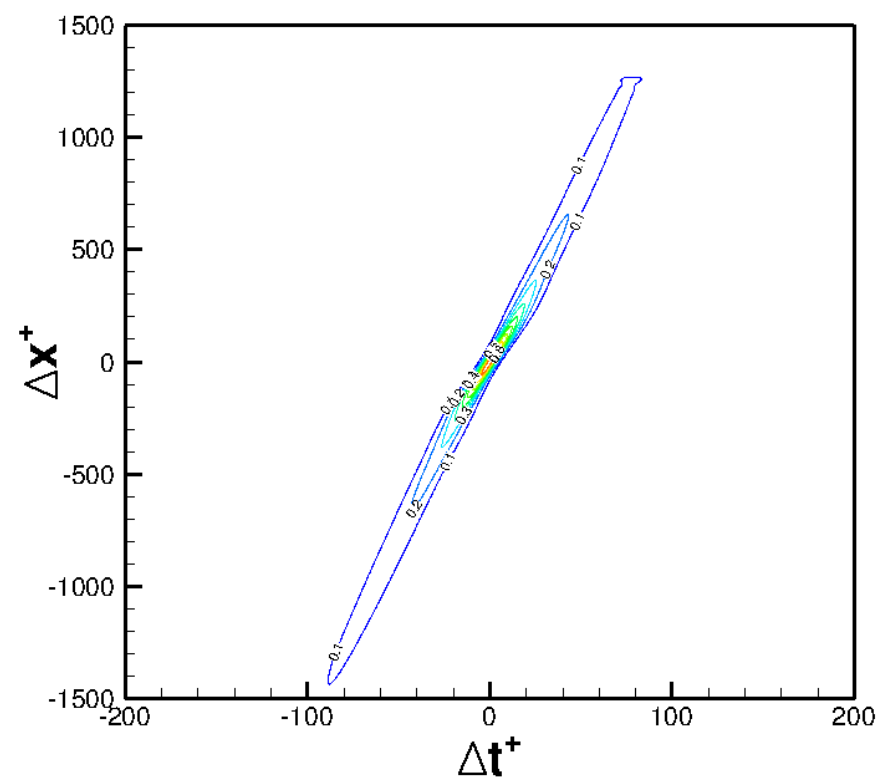

(a) Wall

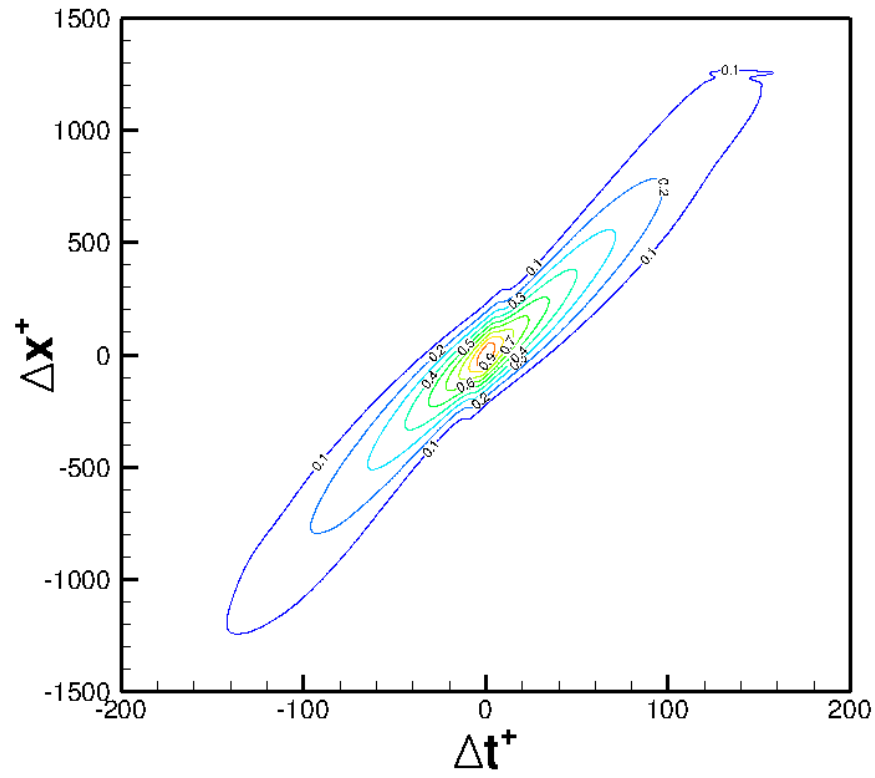

(b) Freestream

Figure 8. Space-time correlation coefficient of the pressure signal at selected heights for the Mach 2.5 DNS. Contour levels vary from 0.1 to 0.9 with increments of 0.1 . 


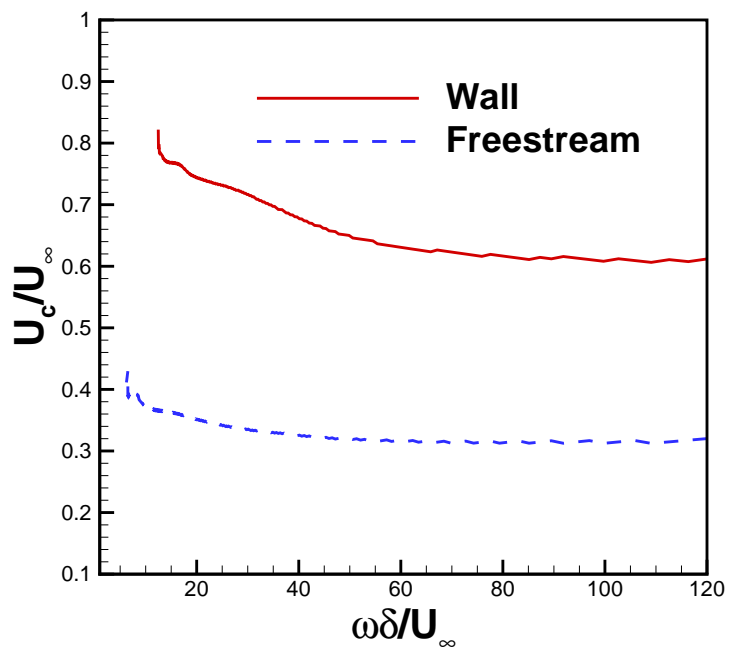

Figure 9. Convection velocity of the pressure fluctuation as a function of frequency at selected heights.

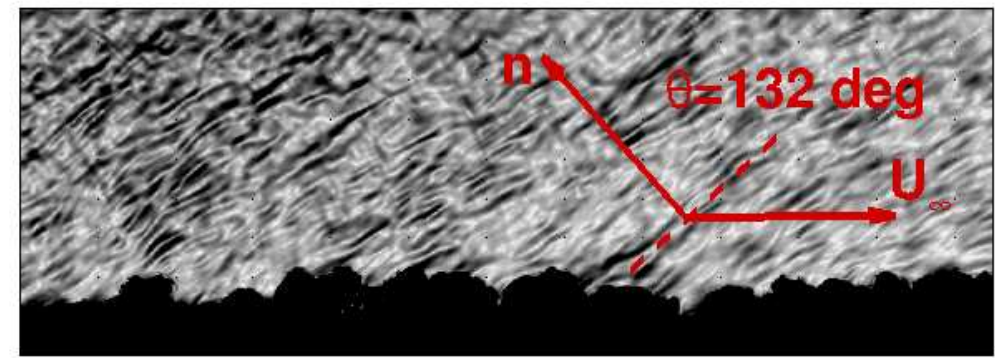

Figure 10. Numerical Schlieren image based on instantaneous flow field within streamwise wallnormal plane for the Mach 2.5 DNS. Contour levels are selected to emphasize disturbances in the freestream. The red dashed line is approximately aligned with the wave front and $n$ is the normal to the wave front; $\theta$ is the angle between the flow direction and $n$. 


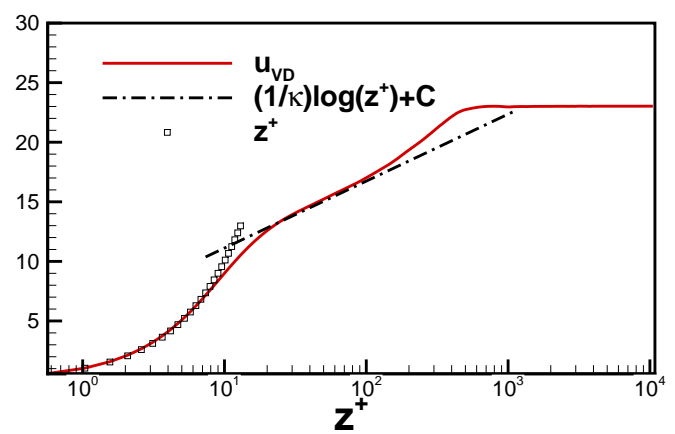

Figure 11. Van-Driest transformed mean velocity profile for the Mach 6 DNS $(\kappa=0.41, C=5.5)$.

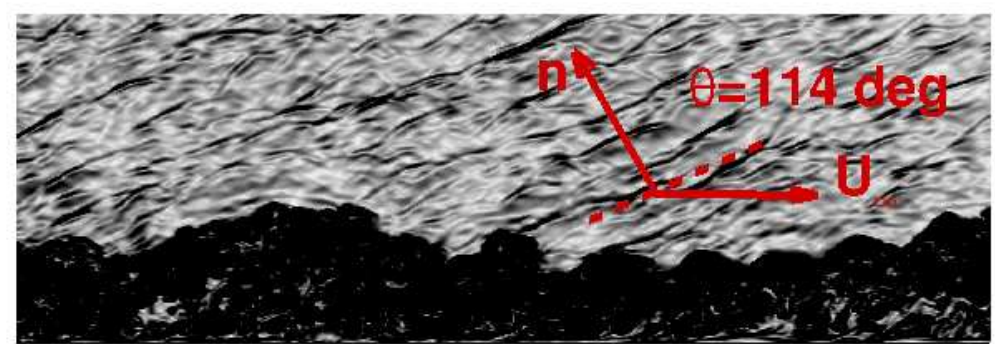

Figure 12. Numerical Schlieren image based on instantaneous flow field within streamwise wallnormal plane for the Mach 6 DNS. Contour levels are selected to emphasize disturbances in the freestream. The red dashed line is approximately aligned with the wave front and $n$ is the normal to the wave front; $\theta$ is the angle between the flow direction and $n$. 